\title{
Adaptive Iteration Stopping Criterion for AMSS Equations
}

\author{
LI Chang-le, LIU Gang-feng* and ZHAO Jie \\ State Key Laboratory of Robotics and System, \\ Harbin Institute of Technology, Harbin, P. R. China \\ *lgf1216@163.com
}

\begin{abstract}
When AMSS (Affine Morphological Scale Space) operator is applied in image filtering, the scale parameter $t$ has great impact on the filtering results. In order to determine the parameters more precisely, this paper analyzed affine invariance properties and classical invariance properties of AMSS operator using the affine transform theory And the numerical solution of AMSS equation is realized with the finite difference method . Based on the cross entropy theory, the forms of cross entropy under the two situations of standard image and nonstandard image were analyzed. The relationship between cross entropy and the scale parameter was explored in both cases, and the AMSS equation's iteration stopping time is determined based on this criterion. Experiment shows that the satisfactory smooth images can be achieved based on the method of cross entropy iteration stopping, under the circumstances of both standard and nonstandard images.
\end{abstract}

Keywords_partial differential equation; AMSS equation; cross entropy; image filter; surface defects

\section{INTRODUCTION}

Image processing based on the partial differential equation has become a hotspot in recent years [1, 2]. It is widely applied to imagine filtering, imagine restoration, imagine segmentation and imagine enhancement, and performs better than the traditional imagine processing methods. It has developed into an important branch in the field of imagine processing. The partial differential equation, which originates from calculus of variations and deformable model, is an important tool especially in imagine filtering, an area of imagine preprocessing [3, 4]. Image denoising method under partial differential equation is to solve a partial differential equation with the original image as the initial condition, and take the solution as the results after filtering.

Image filtering processing belongs to the field of image pretreatment, which needs to satisfy two conditions: contrast invariance and affine invariance at the same time. AMSS equation is one of the few partial differential equations that can satisfy the two conditions simultaneously. However, in the process of achieving the numerical solution of AMSS equation, the AMSS equation's iteration stopping time, the scale parameter $t$ can greatly influence the filtering results. If stopping time $t$ is too large, it will make image too smooth. Otherwise it will lead to the unsatisfactory filtering results. In the past, while making sure the parameter was determined by

This work is supported by National High-tech R \& D Program of China (No.2015BAF10B02, No.2014BAF12B06, and No.2014AA041601) and the project of the State Key Laboratory of Robotics and System (SKLRS201410B picking up ideal $t$ artificially based on the multiple scale parameter results. Therefore, it limits the practical application of AMSS equation, and affects AMSS operator filtering results as well.

\section{AFFINE INVARIANT MORPHOLOGICAL EQUATION}

\section{A. Affine transformation}

The essence of affine transformation is conversion among coordinate systems [5, 6]. In theory, image affine conversion doesn't change the content of the image . It refers to the relation between two images formed by the same object. In other words, it relates to the transformation of two image pixel coordinates. Firstly, the affine coordinate system is shown in figure 1 to give affine transformation a brief introduction.
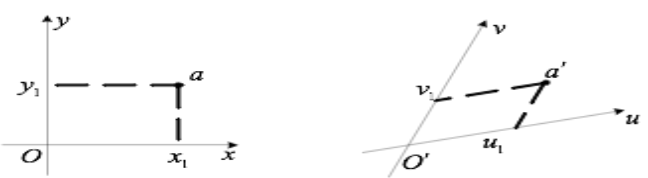

Fig. 1. Cartesian coordinates and affine coordinates

To the two images of the same object it equals to two coordinate systems, and the similar definition of image affine transformation can be got:

$$
f_{1}(x, y)=Q f_{2}(x, y)+B
$$

In the equation, $f_{1}(x, y), f_{2}(x, y)$ indicate two images of the same object, and separately satisfy $|\mathrm{Q}| \neq 0$.

\section{B. Affine invariant morphological operator}

The basic idea of morphological operators is to measure and extract the corresponding forms in the image with certain morphology structure element to achieve the purpose of image analysis and recognition. Under the scale $t$, expansion and erosion operators are defined as:

$$
\begin{aligned}
& D_{t} f(x, y)=\sup _{\left(x^{\prime}, y^{\prime}\right) \in t G} f\left(x-x^{\prime}, y-y^{\prime}\right) \\
& E_{t} f(x, y)=\inf _{\left(x^{\prime}, y^{\prime}\right) \in t G} f\left(x+x^{\prime}, y^{+}+y^{\prime}\right)
\end{aligned}
$$

In the equation $G$ is a structure element. $D_{t}$ is an expansion operator in scale parameter $t$, and $E_{t}$ is corrosion operator in scale parameter. 
According to operators' differential property the following partial differential equation can be inferred by means of localization and iteration [7-9].

$$
\frac{\partial \mathrm{f}}{\partial \mathrm{t}}=|\mathrm{Df}|(\operatorname{curv}(\mathrm{f}))^{1 / 3}
$$

Equation (4) is an AMSS equation, whose solution at moment $t$ can be used as the result of the image filtering .

\section{Numerical solution of AMSS operator}

In the practical application of the AMSS equation, it is rare to get the analytical solution of the equation. Since the strip surface defect images are interval sampled discrete digital images in two-dimensional space, the bisection grid needed by finite difference method is naturally formed . Therefore, the finite difference method is adopted to achieve the numerical calculation of AMSS equation.

The image $f_{0}(x, y)$ is taken as the initial value at the zero moment of (4), and then the solution of the moment $t$ can be used as the result of the image filtering. The mathematical

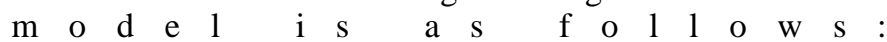

$$
\left\{\begin{array}{c}
\frac{\partial \mathrm{f}}{\partial \mathrm{t}}=|\mathrm{Df} t|(\operatorname{curv}(\mathrm{f}))^{1 / 3} \\
\mathrm{f}(\mathrm{x}, \mathrm{y}, 0)=\mathrm{f}_{0}
\end{array}\right.
$$

According to the basic ideas of the finite difference method, the partial derivative of the variable is approximated by the ratio of the difference between the two adjacent points with finite distance and the distance between the two points [10]. The left part of (5) can be replaced and derived with the difference form $\frac{\mathrm{f}_{\mathrm{t}+\Delta t} \mathrm{f}_{\mathrm{t}}}{\Delta \mathrm{t}}$, and the following can be obtained:

$$
\left\{\begin{array}{c}
\frac{\mathrm{f}_{\mathrm{t}+\Delta t}-\mathrm{f}_{\mathrm{t}}}{\Delta \mathrm{t}}=|\mathrm{Df}|\left(\operatorname{curv}\left(\mathrm{f}_{\mathrm{t}}\right)\right)^{1 / 3} \\
\mathrm{f}(\mathrm{x}, \mathrm{y}, 0)=\mathrm{f}_{0}
\end{array}\right.
$$

$D f_{t}$ in the formula is the gradient of $f_{t}$, and $\operatorname{curv}\left(f_{t}\right)$ is the curvature.

According to the finite difference theory, the central difference scheme of first order partial derivative is:

$$
\left(f_{x}\right)_{i, j}=\frac{f_{i+1, j}-f_{i-1, j}}{2 h}
$$

The difference scheme of second order partial derivative is:

$$
\begin{gathered}
\left(f_{x x}\right)_{i, j}=\frac{f_{i+1, j}+f_{i-1, j}-f_{i, j}}{h^{2}} \\
\left(f_{x y}\right)_{i, j}=\frac{f_{i+1, j+1}+f_{i-1, j-1}-f_{i-1, j+1}-f_{i+1, j-1}}{4 h^{2}}
\end{gathered}
$$

Likewise, the difference scheme of $f_{y}$ is similar to $f_{x}$, and that of $f_{y y}$ is similar to $f_{x x}$. So the solution of (8) can be calculated, and the results of image filtering using the AMSS operator are also obtained.

\section{ADAPTIVE ITERATIVE STOPPING METHOD}

\section{A. Cross entropy}

Cross entropy is the key indicator in evaluating differences between two images, which can directly reflect the differences between the two images [11, 12]. Through the cross entropy of filtered image and the original image, the differences between the original image and the filtered image can be got. The smaller difference shows the greater similarities between the two images. For the two images, the definition of the cross entropy is:

$$
\operatorname{Hc}\left(f, f_{\mathrm{t}}\right)=\sum_{\mathrm{i}=1}^{\mathrm{n}} \mathrm{q}\left(\beta_{\mathrm{i}}\right) \log _{2}\left(\frac{\mathrm{q}\left(\beta_{\mathrm{i}}\right)}{\mathrm{p}\left(\mathrm{\alpha}_{\mathrm{i}}\right)}\right)
$$

$\mathrm{q}\left(\beta_{\mathrm{i}}\right)$ is the gray distribution of the original image, and $\mathrm{p}\left(\alpha_{\mathrm{i}}\right)$ is the gray distribution of filteredimage. In calculating the symmetric cross entropy, the symmetric form of cross entropy is used to eliminate the influence of $\mathrm{q}\left(\beta_{\mathrm{i}}\right)>\mathrm{p}\left(\alpha_{\mathrm{i}}\right)$ or $\mathrm{q}\left(\beta_{\mathrm{i}}\right)<\mathrm{p}\left(\alpha_{\mathrm{i}}\right)$ on the calculation results.

\section{B. Analysis on the iterative stopping criterion of cross entropy}

Based on AMSS operator filtering method, filtered image gradually becomes smooth with the increase of the scale parameter $t$, , and it deviates from the original image. In the experiment, if synthetic noise images with the properties of standard images are used, the cross entropy between the two images can be got from the gray distribution of the original image and the filtered image according to the definition of the cross entropy. The scale parameters of minimum cross entropy can be found according to different scale parameters, , and the AMSS operator iteration stopping time is determined. However, in practical application, most of the images from the image acquisition system are not the standard original image. Therefore, in the actual engineering application, the foregoing form cannot be used directly to determine the cross entropy iteration stopping time. The form of cross entropy is modified in this paper to fit practical engineering applications. The modification of cross entropy is defined as follows:

When the scale parameter is $t$, the filtering result is $f_{t}$. $f_{0}$ is used to represent the original image, and $e_{t}=f_{0}-f_{t}$, can be obtained. If $\mathrm{f}_{\mathrm{t}}$ there is the no noise standard image for $\mathrm{f}_{0}$, then the information contained in $e_{t}$ is all noise information, while the filtering result $f_{t+\Delta t}$ is a smooth transition images when the scale parameter is $(t+\Delta t)$ the filtering result $f$ is a smooth transition of images, and the information contained in $\mathrm{e}_{\Delta t}=\mathrm{f}_{\mathrm{t}}-\mathrm{f}_{\mathrm{t}+\Delta \mathrm{t}}$ is the image information. According to the definition of cross entropy, the cross entropy of $e_{t}$ and $e_{\Delta t}$ can be calculated. and the cross entropy defined by the filtered image and the standard image will be transformed into the cross entropy defined by the two difference images. ts specific definition form is:

$$
\left(\operatorname{Hc}\left(\mathrm{e}_{\mathrm{t}}, \mathrm{e}_{\Delta \mathrm{t}}\right)\right)_{\mathrm{t}}=\sum_{\mathrm{i}=1}^{\mathrm{n}} \mathrm{q}\left(\mathrm{e}_{\mathrm{t}, \mathrm{i}}\right) \log _{2}\left(\frac{\mathrm{q}\left(\mathrm{e}_{\mathrm{t}, \mathrm{i}}\right)}{\mathrm{p}\left(\mathrm{e}_{\Delta t, \mathrm{i}}\right)}\right)
$$

In the formula, $q\left(e_{t, i}\right)$ and $p\left(e_{\Delta, i}\right)$ is the gray distribution ofare the gray distribution of $e_{t}$ and $e_{\Delta t}$ respectively.

But in the specific calculation only image $\mathrm{f}_{t}$ can be got as the best approximation of the actual image $\mathrm{f}_{0}$. Therefore, only the maximum value ( $\mathrm{Hc})_{\mathrm{t}}$ can be obtained, that is, at this time the most noise information is contained in $e_{t}$, and most of the information contained in $\mathrm{e}_{\Delta \mathrm{t}}$ is the image information, which can be used as the basis for the stop of AMSS operator iteration. 


\section{EXPERIMENT AND ANALYSIS}

\section{A. Standard image experiments}

Gaussian noise with mean 0 and variance 0.002 is added to the standard images. The artificially noise added image is filtered, and peak signal-to-noise ratio PSNR and mean square error EMS were taken as the evaluation index, which were compared with the traditional filtering algorithm. In Figure 2, (a) is the standard image without noise, (b) is the image added noise, (c) is AMSS operator filtering results of the adaptive iterative stop method, (d) is weighted mean filtering results, $(\mathrm{E})$ is arithmetic mean filtering results, and (f) is the median operator processing results.
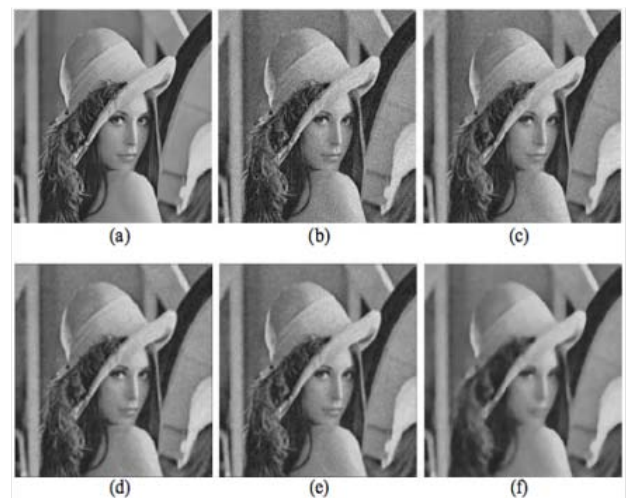

Fig. 2. Comparison of filtering results

Table 1. Evaluating indicator

\begin{tabular}{|l|l|l|}
\hline $\begin{array}{c}\text { Label in } \\
\text { graph }\end{array}$ & \multicolumn{1}{|c|}{ PSNR } & \multicolumn{1}{|c|}{ EMS } \\
\hline (c) & 67.9667 & 72.6651 \\
\hline (d) & 66.6485 & 78.0139 \\
\hline (e) & 64.1511 & 106.4222 \\
\hline (f) & 59.7614 & 165.0723 \\
\hline
\end{tabular}

Seen from the objective evaluation index in Table 1,in the filtering experiment using standard image synthetic noise, the peak signal-to-noise PSNR value of (c) in Figure 2is relatively large, and the mean square error EMS value is relatively small, which illustrates that (c) in Figure 2contains less noise, and its difference from the standard image is small. The corresponding peak signal-to-noise ratio of (d) - (e) in Figure 2 is small, while the mean square difference is relatively large It shows that the deviation of the three images from the standard image is great, and the filtering effect is relatively poor. The objective evaluation index in Table 1 proves that the effect of the algorithm designed in the paper is better than the traditional image filtering algorithm, which is consistent with subjective observation results of 2 .

The synthetic noise image (b) is fitered with different scale parameters $t$ to verify monopole characteristics of cross entropy. As shown in Figure 3, the abscissa is parameter $t$, and the ordinate is cross entropy $\mathrm{Hc}$.

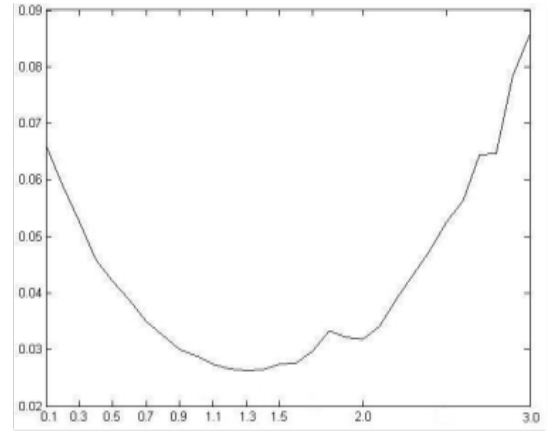

Fig. 3. Relationship between cross entropy Hc and parameter t

Figure 3 shows changing trail of cross entropy with the changes of parameter t from 0.1 to 3 ,, from which we can see that when $t=1.3$ the cross entropy is minimized, and the filtering results is close to the original image. Moreover, when the parameter $t$ gradually increased, the cross entropy value grows accordingly. It shows the difference between the filtered image and the standard image enlarges, and if the parameter $t$ selected is too large, the resulting image will be too smooth.

\section{B. Real image experiments}

In the actual engineering application, standard image without noise and corresponding to the actual image cannot be achieved, and the objective evaluation indexes, such as peak signal-to-noise ratio and mean square error which need standard image information, are unable to use. Thus, the evaluation indexes: average gradient $\overline{\text { grad }}$ and information entropy $\mathrm{H}$, which do not need the standard image information are selected in the experiment. The average gradient reflects the image clarity, and the information entropy illustrates the amount of information in the image. In the experiment the common scratch defect of strip surface is selected to process. Fig.4 shows the curve relation between the scale parameter $t$ and cross entropy $(\mathrm{Hc})_{t}$, where the abscissa is $t$, and the ordinate is $(\mathrm{Hc})_{t}$. When $t<0.6$, the value of cross entropy (Hc) gradually increased, and the difference between $e_{t}$ and $e_{\Delta t}$ expanded in the process. When $t=0.6$, the cross entropy (Hc) reaches the maximum value, which shows the greatest difference between $e_{t}$ and $e_{\Delta t}$. is the maximum, The filtered image $f_{t}$ obtained by this parameter is the optimal filtering result of the actual image. And when $t>0.6$, the cross entropy showed a decreasing trend, indicating that the same image information shared by the two images $e_{t}$ and $e_{\Delta t}$ increased, and they approached each other. Therefore, $t=0.6$ is selected as the scale parameter of the AMSS operator.

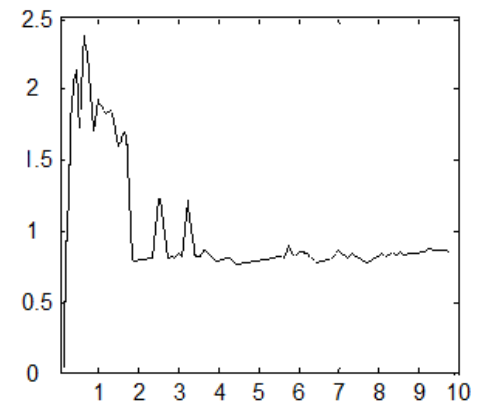

Fig. 4. Relationship between cross entropy (Hc) and parameter t 
Fig. 5 is the comparison between the algorithm proposed and the traditional filtering algorithm. Fig.5 (a) is the image acquisition of scratch defects. Fig. 5 (b) is the filtering results when $\mathrm{t}=0.6$ according to the algorithm presented in this article. Fig.5 (c) shows the arithmetic mean filtering results, Fig.5 (d) is weighted mean filter results. Fig.5 (e) reflects the median operator processing results.

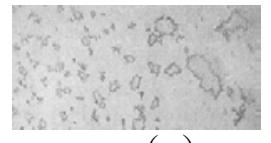

(a)

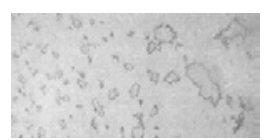

(c)

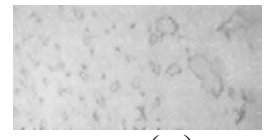

(e)

Fig. 5. Comparison of filtering results

Table 2. Evaluating indicator

\begin{tabular}{|l|l|l|}
\hline $\begin{array}{c}\text { Label in } \\
\text { graph }\end{array}$ & \multicolumn{1}{|c|}{ H } & \multicolumn{1}{|c|}{ grad } \\
\hline (a) & 7.0077 & 8.6633 \\
\hline (b) & 6.9742 & 6.1170 \\
\hline (c) & 6.5215 & 3.0748 \\
\hline (d) & 6.0268 & 2.8304 \\
\hline (e) & 5.8907 & 0.8895 \\
\hline
\end{tabular}

From the subjective observation of the filtering results of each algorithm in Figure 5, it can be found that the processing effect of the algorithm proposed is better than that of the traditional filtering method. From the objective evaluation indexes in Table 2, we can know that the information entropy and average gradient values of the filtering results obtained by the adaptive iterative stopping method, are relatively large, which indicats that the image is more clear, contains more image information. , the average gradient and information entropy of image processed under the traditional filtering method is relatively small, which proves that more detail information of the image will lose in the approach, resulting in sharpness decline of the image. The data in Table 2 directly verify the results of the subjective observations in Figure 5.

\section{CONCLUSION}

This paper fully discussed the theory of AMSS equation, and puts forward the method of stopping cross entropy based on adaptive iterative. Satisfactory filtering effect based on AMSS operator can be obtained through this method. By analyzing specific forms of cross entropy in the synthetic noise image filtering, the cross entropy realization form of the actual image filtering is proposed to adapt to the practical engineering application without standard images. According to the experiment, this method can not only gain satisfactory effects in processing the synthetic noise image, but also is effective in the actual image filtering. The adaptive iterative stopping method based on cross entropy can timely stop the iterative algorithm of AMSS operator, and determine the scale parameter of AMSS operator to achieve better filtering effect. This method provides basis for AMSS operator in real engineering application, and a new feasible method for the pretreatment of the strip surface defect image in production.

\section{ACKNOWLEDGMENT}

This work is supported by National High-tech R \& D Program of China (No.2015BAF10B02, No.2014BAF12B06, and No. 2014AA041601) and the project of the State Key Laboratory of Robotics and System (SKLRS201410B).

\section{REFERENCES}

[1] Tschumperle David, Deriche Rachid. Vector-Valued image regularization with PDEs: A common framework for different applications[J]. IEEE transactions on pattern analysis and machine intelligence, 2005,27(4): 506-517.

[2] Zhang J, Wei Z, Liang X. Adaptive Fractional-order Multi-scale Method for Image De-noising[J]. Journal of Mathematical Imaging \& Vision, 2011, 43(1):39-49.

[3] Yang W, Wei G W, Yang S. Mode Decomposition Evolution Equations[J]. Journal of Scientific Computing, 2012, 50(3):495-518. .

[4] Åström F, Baravdish G, Felsberg M. On Tensor-Based PDEs and Their Corresponding Variational Formulations with Application to Color Image Denoising[J]. Lecture Notes in Computer Science, 2012, 7574(1):215-228.

[5] Tao C, Yi S U, Jiang Y M, et al. Affine Invariant Feature Extraction Based on Affine Geometry[J]. Journal of Image \& Graphics, 2007, 12(9):1633-1641.

[6] Brais Martinez, Xavier Binefa. Piecewise affine kernel tracking for nonplanar targets [J]. Pattern Recognition, 2008, 41(12): 3682-3691

[7] Gilles Aubert, Lumminita Vese. A variational method in image recovery[J]. SIAM J. NUMER.ANAL, 1997,34(5) : 1948-1979.

[8] Alvarez L, Guichard F, Lions P L, et al. Axioms and fundamental equation of image processing[J]. Archive for rational Mechanics and Analysis, 1993,123(3) : 199-257.

[9] Jia, Liu, Caicheng, et al. Image Denoising Based on BEMD and PDE[C]// 2011. 2011:110 - 112.

[10] Matsue K. Effects of Corporate Social Responsibility on Corporate Financial Performance: A Competitive-Action Perspective[J]. Journal of Management, 2015, 1616.

[11] S. Suresh, N. Sundararajan, P. Saratchandran. Risk-sensitive loss functions for sparse multi-category classification problems [J]. Information Sciences, 2008, 178(12): 2621-2638.

[12] YIN Peng-Yeng. Multilevel minimum cross entropy threshold selection beasd on particle swarm optimization [J]. Applied Mathematics and Computation, 2007, 184(2): 503-513. 